\title{
New approach for structural behavior of variables
}

\author{
Abdul Hamid Ganie \\ Basic Science Department, College of Science and Theoretical Studies, Saudi Electronic University, Abha Male 61421, Kingdom of \\ Saudi Arabia.
}

\begin{abstract}
The main scenario of the present paper is to introduce certain approach of variables by setting the structural behavior of fractional inequalities. Some new structural properties will be established concerning them.
\end{abstract}

Keywords: Fractional notion, variables, Lebesgue measurable functions.

2020 MSC: 26D53, 26A33, 60E15.

(C)2021 All rights reserved.

\section{Introduction}

In recent years, the importance of integral inequalities play a vital appearance in the mathematics, sciences and technology (MST). It has achieved a significant development from last few decades and can be seen in $[1,16,19]$ and many others. The structure of fractional calculus has a first hand knock over the area presuming the way out of various tools in MST; in order to attract more closeness towards the subject and to determine its utility, various kinds of statements and uses of fractional derivatives have been expanded. It was Riemannan Liouville and Letnikov who are most familiar in this direction of study as can be seen in $[5,9-11,15,18,20,22]$, and etc.

It was Caputo who put together the classical sense of the Riemannan Liouville fractional derivative for solving fractional differential equations with applied approaches called initial conditions. Further Letnikov has synthesized the notion of fractional calculus given by Leibniz in a new way.

Quite recently, the author in [8] introduced new notion on probability theory by involving fractional calculus in to play and extended the classical results. More structures and its various applications to real world problems which includes differential equations, fluid mechanics, biomathematics etc can be found in $[2,3,7,14,24]$, and many others. We now present some definitions as follows.

Definition $1.1([16,17,21])$. We define Riemann Liouville fractional integral of order $\kappa \geqslant 0$ for a continuous map $\mathfrak{g}(v)$ on $[c, d]$, which is given by

$$
\mathcal{R}_{\mathbf{a}}^{\kappa}[\mathfrak{g}(v)]=\frac{1}{\Gamma(\kappa)} \int_{a}^{v}(v-s)^{\kappa-1} \mathfrak{g}(s) d s \text { for } a<v \leqslant d,
$$

where $\Gamma(\kappa)=\int_{0}^{\infty} e^{-v} v^{\kappa-1} d v$ is called as the Gamma function and $\mathcal{J}_{\mathfrak{a}}^{0}={ }_{+} \mathcal{J}_{\mathfrak{a}}^{0} \mathfrak{g}(\nu)=\mathfrak{g}(v)$.

Email address: a.ganie@seu.edu.sa (Abdul Hamid Ganie)

doi: $10.22436 /$ jnsa.014.05.05

Received: 2020-10-21 Revised: 2020-11-23 Accepted: 2021-01-18 
It is important to see that for $\kappa \geqslant 0$, we have following well known properties:

$$
\mathcal{R}_{\mathfrak{c}}^{\kappa} \mathcal{R}_{\mathfrak{c}}^{\omega}[\mathfrak{g}(v)]=\mathcal{R}_{\mathfrak{c}}^{\kappa+\omega}[\mathfrak{g}(v)]
$$

and

$$
\mathcal{R}_{\mathfrak{c}}^{k} \mathcal{R}_{\mathfrak{c}}^{\omega}[\mathfrak{g}(v)]=\mathcal{R}_{\mathfrak{c}}^{\omega} \mathcal{R}_{\mathfrak{c}}^{k}[\mathfrak{g}(\nu)] .
$$

Definition 1.2 ([25]). For $k \geqslant 0$ and $1 \leqslant p<\infty$, define $\mathfrak{L}_{p, k}(c, d)$ by

$$
\|\mathfrak{g}\|_{\mathfrak{L}_{\mathfrak{p}, \mathrm{k}}(\mathrm{c}, \mathrm{d})}=\left[\int_{\mathfrak{c}}^{\mathrm{d}}|\mathfrak{g}(v)|^{p} v^{k} \mathrm{~d} v\right]^{\frac{1}{\mathfrak{p}}}<\infty
$$

which is called as Lebesgue measurable real-valued maps $\mathfrak{g}$ on $[\mathrm{c}, \mathrm{d}]$.

Definition 1.3 ([25]). For $c \in R$ and $1 \leqslant p<\infty$, define $\mathfrak{X}_{\mathfrak{c}}^{\mathfrak{p}}(c, d)$ by

$$
\|\mathfrak{g}\|_{\mathfrak{X}_{\mathfrak{c}}^{\mathfrak{p}}}=\left[\int_{\mathcal{c}}^{\mathrm{d}}\left|\nu^{\mathfrak{c}} \mathfrak{g}(v)\right|^{p} \frac{\mathrm{d} v}{v}\right]^{\frac{1}{\mathfrak{p}}}<\infty
$$

called as the real-valued Lebesgue measurable functions $\mathfrak{g}$ on $[c, d]$ and for the choice of $p=\infty$, consider it as

$$
\|\mathfrak{g}\|_{\mathfrak{X}_{\mathfrak{c}}^{\infty}}=\operatorname{ess} \sup _{\mathcal{c} \leqslant \nu \leqslant d}\left|\nu^{\mathfrak{c}} \mathfrak{g}(v)\right|
$$

Remark 1.4. By choosing $\mathrm{c}=\frac{(\mathfrak{u}+1)}{\mathrm{p}}$ with $1 \leqslant p<\infty, \mathfrak{u} \geqslant 0$, then $\mathfrak{X}_{\mathfrak{c}}^{\mathfrak{p}}(\mathrm{c}, \mathrm{d})$ coincides to $\mathfrak{L}_{\mathfrak{p}, \mathrm{k}}(\mathrm{c}, \mathrm{d})$ and also for $c=\left(\frac{1}{p}\right)$ with $1 \leqslant p<\infty$, then $\mathfrak{X}_{\mathfrak{c}}^{p}(c, d)$ is reduced to the classical space $\mathfrak{L}^{p}(c, d)$.

Definition 1.5. ([25]) Let $\mathfrak{g} \in \mathfrak{L}_{1, \mathrm{~s}}$ with $k \geqslant 0$. The generalized Riemann-Liouville fractional integrals ${ }_{+} \mathcal{R}_{\mathbf{c}, \mathfrak{h}}^{\kappa, s}$ and $\_\mathcal{R}_{c, \mathfrak{h}}^{\kappa, s}$ with order $\kappa \geqslant 0, \mathfrak{h}>0$ are given by

$$
\mathcal{R}_{c, \mathfrak{h}}^{\kappa, s} \mathfrak{g}(u)=\frac{(s+1)^{1-\frac{k}{\mathfrak{h}}}}{\mathfrak{h} \Gamma(\kappa)} \int_{\mathfrak{c}}^{u}\left(u^{s+1}-v^{s+1}\right)^{\frac{\kappa}{h}-1} v^{s} \mathfrak{g}(v) d v \text { for } u>c
$$

Throughout the paper, the random variable $X$ will be abbreviated by r.v..

Definition 1.6. For a r.v. $X$ having a positive p.d.f. $\mathfrak{g}$ on $[c, d]$, then, for $s \geqslant 0$ and $c<v \leqslant d$, the fractional expectation function with order $\mathrm{K}$ is given as

$$
E_{X, k}^{\mathfrak{h}}(\mathfrak{u})={ }_{+} \mathcal{R}_{c, \mathfrak{h}}^{\kappa, s}[u \mathfrak{g}(u)]=\frac{(s+1)^{1-\frac{k}{\mathfrak{h}}}}{\mathfrak{h} \Gamma(\kappa)} \int_{\mathfrak{c}}^{\mathfrak{u}}\left(\mathfrak{u}^{s+1}-v^{s+1}\right)^{\frac{\kappa}{\mathfrak{h}}-1} v^{s+1} \mathfrak{g}(v) d v
$$

Definition 1.7. We define the fractional expectation of positive real valued function of order $\mathrm{k}$ for a r.v. $X-E(X)$ as

$$
E_{X-E(X), \kappa}^{\mathfrak{h}}(u)=\frac{(s+1)^{1-\frac{k}{h}}}{\mathfrak{h} \Gamma(\kappa)} \int_{c}^{u}\left(u^{s+1}-v^{s+1}\right)^{\frac{k}{h}-1}(t-E(X)) v^{s} \mathfrak{g}(v) d v .
$$

It is important to note that for $u=d$, the above definitions take the following form.

Definition 1.8. For a r.v. $X$ having a positive p.d.f. $\mathfrak{g}$ on $[c, d]$. For $s \geqslant 0$, the fractional expectation function of order $\mathrm{k}$ is given as

$$
E_{X, k}^{\mathfrak{h}}(u=d)=\frac{(s+1)^{1-\frac{k}{h}}}{\mathfrak{h} \Gamma(\kappa)} \int_{c}^{d}\left(d^{s+1}-v^{s+1}\right)^{\frac{k}{h}-1} v^{s+1} \mathfrak{g}(v) d v
$$


Definition 1.9. Let $E(X)$ represents the mathematical expectation of $X$ be a r.v. with a positive p.d.f. $\mathfrak{g}$ defined on $[c, d]$. Then, for $s \geqslant 0$ and $c<v \leqslant d$, we define the fractional variance function of order $k$ as

$$
\sigma_{X, k, \mathfrak{h}}^{2}(u)=+\mathcal{R}_{c, \mathfrak{h}}^{\kappa, s}\left[(u-E(X))^{2} f(u)\right]=\frac{(s+1)^{1-\frac{k}{h}}}{\mathfrak{h} \Gamma(\kappa)} \int_{\mathfrak{c}}^{u}\left(u^{s+1}-v^{s+1}\right)^{\frac{k}{h}-1}(v-E(X))^{2} v^{s} \mathfrak{g}(v) d v
$$

Definition 1.10. If $u=d$, then we define the fractional variance function of order $k$ as

$$
\sigma_{X, k, \mathfrak{h}}^{2}(u=d)=\frac{(s+1)^{1-\frac{k}{\mathfrak{h}}}}{\mathfrak{h} \Gamma(\kappa)} \int_{c}^{d}\left(d^{s+1}-v^{s+1}\right)^{\frac{k}{h}-1}(v-E(X))^{2} v^{s} \mathfrak{g}(v) d v
$$

Now choosing $\mathrm{k}$ and $\mathrm{s}$ differently and $\mathrm{s}$, we have the following remark.

Remark 1.11.

(D1) Choosing $s=0$ and $\kappa=1=\mathfrak{h}$ in Definition 1.6, we get $E_{X, 1,1}=E(X)$ as the classical expectation of r.v. $X$.

(D2) Choosing $s=0$ and $\kappa=1=\mathfrak{h}$ in Definition 1.8, we get $\sigma_{X, 1}^{2}=\sigma^{2}(X)=\int_{\mathfrak{c}}^{d}(v-E(X))^{2} \mathfrak{g}(v) d v$ as the classical variance of r.v. $X$.

(D3) Choosing $\mathrm{k}=1$, we get the well known result $\mathcal{R}^{\mathrm{K}}[\mathrm{f}(\mathrm{d})]=1$.

\section{Main results}

This portion will be dealing with the new generalization of results of continuous r.v. with fractional integral order.

Theorem 2.1. For an r.v. X having a positive p.d.f. $\mathfrak{g}$ on $[\mathrm{c}, \mathrm{d}]$, then for all $\mathrm{c}<\boldsymbol{v} \leqslant \mathrm{d}, \mathrm{k} \geqslant 0$ and $\mathrm{s} \geqslant 0$, we have

(i) the inequality

$$
\begin{aligned}
& { }_{+} \mathcal{R}_{\mathfrak{c}, \mathfrak{h}}^{\kappa, s}[g(u)] \sigma_{X, k, \mathfrak{h}}^{2}(u)\left(E_{X-E(X), k}^{\mathfrak{h}}(u)\right)^{2} \\
& \quad \leqslant\|g\|_{\infty}^{2}\left(\frac{(s+1)^{1-\frac{k}{\mathfrak{h}}}\left(u^{s+1}-c^{s+1}\right)^{\frac{k}{h}}}{\Gamma(\kappa+1)}+\mathcal{R}_{c, \mathfrak{h}}^{k, s}\left[u^{2 s+2}\right]-\left({ }_{+} \mathcal{R}_{c, \mathfrak{h}}^{\kappa, s}[u]\right)^{2}\right)
\end{aligned}
$$

holds provided $\mathrm{g} \in \mathrm{L}_{\infty}[\mathrm{c}, \mathrm{d}]$; and

(ii) the inequality

$$
{ }_{+} \mathcal{R}_{c, \mathfrak{h}}^{k, s}[g(u)] \sigma_{X, k, \mathfrak{h}}^{2}(u)\left(E_{X-E \mathfrak{h}(X), k}(u)\right)^{2} \leqslant \frac{1}{2}\left(u^{s+1}-c^{s+1}\right)^{2}\left({ }_{+} \mathcal{R}_{c, \mathfrak{h}}^{k, s}[u]\right)
$$

holds.

Proof. We first consider the function $\mathfrak{H}$ for $v, \mathrm{~m} \in(\mathrm{c}, \mathrm{v}), \mathrm{c}<\boldsymbol{v} \leqslant \mathrm{d}$

$$
\mathfrak{H}(v, \mathrm{~m})=\left(\mathfrak{H}_{1}(v)-\mathfrak{H}_{1}(\mathrm{~m})\right)\left(\mathfrak{H}_{2}(v)-\mathfrak{H}_{2}(\mathrm{~m})\right)
$$

where $\kappa \geqslant 0$. Now multiplying (2.2) by $\frac{(s+1)^{1-\frac{k}{h}}\left(u^{s+1}-v^{s+1}\right)^{\frac{k}{h}-1}}{\mathfrak{h} \Gamma(\kappa)} v^{s} \mathfrak{p}(v)$ on both sides, where the map $\mathfrak{p}$ is $\mathfrak{p}:[c, d] \rightarrow \mathbf{R}^{+}$with $v \in(c, u)$. We now integrate obtained identity from $c$ to $u$, we see

$$
\begin{aligned}
& \frac{(s+1)^{1-\frac{k}{h}}}{\mathfrak{h} \Gamma(\kappa)} \int_{\mathfrak{c}}^{\mathfrak{u}}\left(u^{s+1}-v^{s+1}\right)^{\frac{k}{\mathfrak{h}}-1} \mathfrak{p}(v) \mathfrak{H}(v, m) v^{s} d v \\
& ={ }_{+} \mathcal{R}_{\mathfrak{c}, \mathfrak{h}}^{\kappa, s}\left[\mathfrak{p} \mathfrak{H}_{1} \mathfrak{H}_{2}(v)\right]-\mathfrak{H}_{2}(m)_{+} \mathcal{R}_{\mathfrak{c}, \mathfrak{h}}^{\kappa, s}\left[\mathfrak{p} \mathfrak{H}_{1}(v)\right] \\
& -\mathfrak{H}_{1}(\mathrm{~m})_{+} \mathcal{R}_{\mathrm{c}, \mathfrak{h}}^{\mathrm{k}, \mathrm{s}}\left[\mathfrak{p} \mathfrak{H}_{2}(v)\right]+\mathfrak{H}_{1}(\mathrm{~m}) \mathfrak{H}_{2}(\mathrm{~m})_{+} \mathcal{R}_{\mathfrak{c}, \mathfrak{h}}^{\mathrm{k}, \mathrm{s}}[\mathfrak{p}(\mathrm{u})]
\end{aligned}
$$


Now multiplying (2.3) by $\frac{(s+1)^{1-\kappa}\left(u^{s+1}-m^{s+1}\right)^{\frac{k}{h}-1}}{\mathfrak{h} \Gamma(\kappa)} \mathfrak{p}(m) m^{s}$ for $m \in(c, u)$, and then integrating over $(c, u)$ with respect to $m$, we see

$$
\begin{aligned}
& \frac{(s+1)^{2-2 \kappa}}{\mathfrak{h}^{2} \Gamma^{2}(\kappa)} \int_{c}^{\mathfrak{u}} \int_{c}^{u}\left(u^{s+1}-v^{s+1}\right)^{\frac{\kappa}{\mathfrak{h}}-1}\left(u^{s+1}-m^{s+1}\right)^{\frac{\kappa}{\mathfrak{h}}-1} \mathfrak{p}(v) \mathfrak{p}(\mathrm{m}) \mathfrak{H}(v, m) v^{s} m^{s} d v d m \\
& =2+\mathcal{R}_{\mathbf{c}, \mathfrak{h}}^{\kappa, s}[\mathfrak{p}(v)]_{+} \mathcal{R}_{\mathbf{c}, \mathfrak{h}}^{\kappa, s}\left[\mathfrak{p} \mathfrak{H}_{1} \mathfrak{H}_{2}(v)\right]-2_{+} \mathcal{R}_{\mathbf{c}, \mathfrak{h}}^{\kappa, s}\left[\mathfrak{p} \mathfrak{H}_{1}(v)\right]_{+} \mathcal{R}_{\mathbf{c}, \mathfrak{h}}^{\kappa, s}\left[\mathfrak{p} \mathfrak{H}_{2}(v)\right] .
\end{aligned}
$$

Now in (2.4), choosing $\mathfrak{p}(v)=g(v)$ and $\mathfrak{H}_{1}(v)=\mathfrak{H}_{2}(v)=v^{s+1}-E(X), v \in(c, d)$, we see

$$
\begin{aligned}
& \frac{(s+1)^{2-2 \kappa}}{\mathfrak{h}^{2} \Gamma^{2}(\kappa)} \int_{c}^{v} \int_{c}^{u}\left(u^{s+1}-v^{s+1}\right)^{\frac{k}{h}-1}\left(u^{s+1}-m^{s+1}\right)^{\frac{k}{h}-1} g(v) g(m)\left(v^{s+1}-m^{s+1}\right)^{2} v^{s} m^{s} d v d m \\
& \quad=2_{+} \mathcal{R}_{c, \mathfrak{h}}^{k, s}[g(u)]+\mathcal{R}_{c, \mathfrak{h}}^{k, s}\left[g(u)\left(u^{s+1}-E(X)\right)^{2}\right]-2\left[+\mathcal{R}_{c, \mathfrak{h}}^{k, s} g(u)\left(u^{s+1}-E(X)\right)^{2}\right] .
\end{aligned}
$$

But on the other hand, we see

$$
\begin{aligned}
& \frac{(s+1)^{2-2 k}}{\mathfrak{h}^{2} \Gamma^{2}(\kappa)} \int_{c}^{u} \int_{c}^{u}\left(u^{s+1}-v^{s+1}\right)^{\frac{k}{\mathfrak{h}}-1}\left(u^{s+1}-m^{s+1}\right)^{\frac{k}{\mathfrak{h}}-1} g(v) g(m)\left(v^{s+1}-m^{s+1}\right)^{2} v^{s} m^{s} d v d m \\
& \quad \leqslant\|g\|_{\infty}^{2}\left(2 \frac{(s+1)^{1-\frac{k}{h}}\left(u^{s+1}-c^{s+1}\right)^{\frac{k}{\mathfrak{h}}}}{\Gamma(k+1)}+\mathcal{R}_{c, \mathfrak{h}}^{k, s}\left[u^{2 s+2}\right]-2\left(+\mathcal{R}_{c, \mathfrak{h}}^{k, s}[u]\right)^{2}\right) .
\end{aligned}
$$

Thus, from (2.5) and (2.6), we get (i) part of this result.

For part (ii), we have

$$
\begin{gathered}
\frac{(s+1)^{2-2 \frac{k}{h}}}{\mathfrak{h}^{2} \Gamma^{2}(\kappa)} \int_{c}^{u} \int_{c}^{u}\left(u^{s+1}-v^{s+1}\right)^{\frac{k}{h}-1}\left(u^{s+1}-m^{s+1}\right)^{k-1} g(t) g(m)\left(v^{s+1}-m^{s+1}\right)^{2} v^{s} m^{s} d v d m \\
\leqslant \sup _{v, m \in[c, u]}\left|\left(v^{s+1}-m^{s+1}\right)\right|^{2}\left[{ }_{+} \mathcal{R}_{c, \mathfrak{h}}^{k, s} g(u)\right]^{2}=\left(u^{s+1}-c^{s+1}\right)^{2}\left[+\mathcal{R}_{c, \mathfrak{h}}^{k, s} g(u)\right]^{2} .
\end{gathered}
$$

Consequently, from (2.5) and (2.7), we get (2.1) as desired.

Corollary 2.2. For $\mathrm{k} \geqslant 0$ and $\mathfrak{h}=1$, choose a continuous r.v. $X$ with p.d.f. $g$ defined in $[c, d]$. Then,

(i) the inequality

$$
\frac{d^{s+1}-c^{s+1}}{\Gamma(\kappa)} \sigma_{X, k, h}^{2}-E_{X, k}^{2} \leqslant\|g\|_{\infty}^{2}\left(\frac{\left(d^{s+1}-c^{s+1}\right)^{2 \kappa+2}}{\Gamma(k+1) \Gamma(\kappa+3)}-\left[\frac{\left(d^{s+1}-c^{s+1}\right)^{k+1}}{\Gamma(k+1)}\right]^{2}\right)
$$

holds if $\mathrm{g} \in \mathrm{L}_{\infty}[\mathrm{c}, \mathrm{d}]$ and $\mathrm{s} \geqslant 0$;

(ii) the inequality

$$
\frac{\mathrm{d}^{\mathrm{s}+1}-\mathrm{c}^{\mathrm{s}+1}}{\Gamma(\mathrm{k})} \sigma_{X, \mathrm{k}, \mathfrak{h}}^{2}-\mathrm{E}_{X, \mathrm{k}}^{2} \leqslant \frac{1}{2}\left(\frac{\left(\mathrm{d}^{\mathrm{s}+1}-\mathrm{c}^{\mathrm{s}+1}\right)^{2 \mathrm{k}}}{\Gamma^{2}(\mathrm{k})}\right)
$$

holds for any $\mathrm{s} \geqslant 0$.

Deduction 2.3. By choosing $\mathrm{k}=1=\mathfrak{h}$ and $\mathrm{s}=0$ in (i) of Corollary 2.2, we get the first part of Theorem 1 in [4].

Deduction 2.4. By choosing $\mathrm{k}=1=\mathfrak{h}$ and $\mathrm{s}=0$ in (ii) of Corollary 2.2, we get the last part of Theorem 1 in [4].

Theorem 2.5. Let $X$ be a r.v. with p.d.f. $\mathrm{g}:[\mathrm{c}, \mathrm{d}] \rightarrow \mathbf{R}^{+}$. Then 
(i) for all $\mathrm{c}<\mathrm{u} \leqslant \mathrm{d}, \mathrm{k} \geqslant 0, \lambda \geqslant 0$ and $\mathrm{s} \geqslant 0$, we have

$$
\begin{aligned}
& { }_{+} \mathcal{R}_{c, \mathfrak{h}}^{k, s}[g(u)] \sigma_{X, \lambda, \mathfrak{h}}^{2}(u)+{ }_{+} \mathcal{R}_{c, \mathfrak{h}}^{\lambda, s}[g(u)] \sigma_{X, k, \mathfrak{h}}^{2}(u)-2\left(E_{X-E(X), k, \mathfrak{h}}(u)\right)\left(E_{X-E(X), \lambda, \mathfrak{h}}(u)\right) \\
& \leqslant\|g\|_{\infty}^{2}\left(\frac{(s+1)^{1-\frac{k}{\mathfrak{h}}}\left(u^{s+1}-c^{s+1}\right)^{\frac{k}{h}}}{\Gamma(\kappa+1)}+\mathcal{R}_{c, \mathfrak{h}}^{\lambda, s}\left[u^{2 s+2}\right]\right) \\
& \quad+\|g\|_{\infty}^{2}\left(\frac{(s+1)^{1-\frac{\lambda}{\mathfrak{h}}}\left(u^{s+1}-c^{s+1}\right)^{\frac{k}{\mathfrak{h}}}}{\Gamma(\lambda+1)}+\mathcal{R}_{c, \mathfrak{h}}^{k, s}\left[u^{2 s+2}\right]-2\left({ }_{+} \mathcal{R}_{c, \mathfrak{h}}^{k, s}[u]\right)\left({ }_{+} \mathcal{R}_{c, \mathfrak{h}}^{\lambda, s}[u]\right)\right)
\end{aligned}
$$

holds for $\mathrm{g} \in \mathrm{L}_{\infty}[\mathrm{c}, \mathrm{d}]$; and

(ii) the inequality

$$
\begin{gathered}
{ }_{+} \mathcal{R}_{\mathbf{c}, \mathfrak{h}}^{\mathrm{k}, \mathrm{s}}[\mathrm{g}(v)] \sigma_{X, \lambda}^{2}(\mathrm{u})+{ }_{+} \mathcal{R}_{\mathbf{c}, \mathfrak{h}}^{\lambda, \mathrm{s}}[\mathrm{g}(\mathrm{u})] \sigma_{X, \mathrm{k}, \mathfrak{h}}^{2}(\mathrm{u})-2\left(\mathrm{E}_{X-\mathrm{E}(X), \mathrm{k}}(\mathrm{u})\right)\left(\mathrm{E}_{X-\mathrm{E}(X), \lambda}(\mathrm{u})\right) \\
\leqslant\left(v^{\mathrm{s}+1}-\mathrm{c}^{\mathrm{s}+1}\right)\left({ }_{+} \mathcal{R}_{\mathbf{c}, \mathfrak{h}}^{\mathrm{k}, \mathrm{s}}[v]\right)\left({ }_{+} \mathcal{R}_{\mathbf{c}, \mathfrak{h}}^{\lambda, \mathrm{s}}[\mathrm{u}]\right)
\end{gathered}
$$

holds for any $\mathrm{c}<\mathrm{u} \leqslant \mathrm{d}, \mathrm{k} \geqslant 0, \lambda \geqslant 0$ and $\mathrm{s} \geqslant 0$.

Proof. From (2.2), we can write

$$
\begin{aligned}
& \frac{(s+1)^{2-\kappa-\lambda}}{\mathfrak{h}^{2} \Gamma(\kappa) \Gamma(\lambda)} \int_{\mathfrak{c}}^{\mathfrak{u}} \int_{\mathfrak{c}}^{\mathfrak{u}}\left(u^{s+1}-v^{s+1}\right)^{\frac{k}{\mathfrak{h}}-1}\left(v^{s+1}-m^{s+1}\right)^{\frac{\kappa}{\mathfrak{h}}-1} \mathfrak{p}(v) \mathfrak{p}(\mathrm{m}) \mathfrak{H}(v, m) v^{s} m^{s} d v d m \\
& ={ }_{+} \mathcal{R}_{\mathfrak{c}, \mathfrak{h}}^{\kappa, s}[\mathfrak{p}(\mathfrak{u})]_{+} \mathcal{R}_{\mathfrak{c}, \mathfrak{h}}^{\lambda, s}\left[\mathfrak{p} \mathfrak{H}_{1} \mathfrak{H}_{2}(u)\right]+{ }_{+} \mathcal{R}_{\mathfrak{c}, \mathfrak{h}}^{\lambda, \mathrm{s}}[\mathfrak{p}(\mathfrak{u})]_{+} \mathcal{R}_{\mathfrak{c}, \mathfrak{h}}^{\kappa, s}\left[\mathfrak{p} \mathfrak{H}_{1} \mathfrak{H}_{2}(u)\right]
\end{aligned}
$$

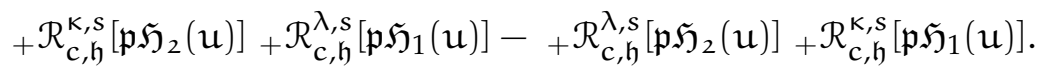

For $u \in(c, d)$, we insert $\mathfrak{p}(u)=g(u), \mathfrak{H}_{1}(u)=\mathfrak{H}_{2}(u)=u^{s+1}-E(X)$ in (2.9), we see

$$
\begin{aligned}
& \frac{(s+1)^{2-\frac{k+\lambda}{\mathfrak{h}}}}{\mathfrak{h}^{2} \Gamma(\kappa) \Gamma(\lambda)} \int_{c}^{u} \int_{c}^{v}\left(u^{s+1}-v^{s+1}\right)^{\frac{k}{\mathfrak{h}}-1}\left(u^{s+1}-m^{s+1}\right)^{\frac{k}{\mathfrak{h}}-1} g(v) g(m)\left(v^{s+1}-m^{s+1}\right)^{2} v^{s} m^{s} d v d m \\
&=+\mathcal{R}_{c, \mathfrak{h}}^{k, s}[g(u)]_{+} \mathcal{R}_{c, \mathfrak{h}}^{\lambda, s}\left[g(u)\left(u^{s+1}-E(X)\right)^{2}\right] \\
&+{ }_{+} \mathcal{R}_{c, \mathfrak{h}}^{\lambda, s}[g(u)]_{+} \mathcal{R}_{c, \mathfrak{h}}^{k, s}\left[g(u)\left(u^{s+1}-E(X)\right)^{2}\right] \\
&-2_{+} \mathcal{R}_{c, \mathfrak{h}}^{k, s}\left[g(u)\left(u^{s+1}-E(X)\right)\right]_{+} \mathcal{R}_{c, \mathfrak{h}}^{\lambda, s}\left[g(u)\left(u^{s+1}-E(X)\right)\right] .
\end{aligned}
$$

But we also see

$$
\begin{aligned}
& \frac{(s+1)^{2-\frac{k+\lambda}{\mathfrak{h}}}}{\mathfrak{h}^{2} \Gamma(\kappa) \Gamma(\lambda)} \int_{\mathfrak{c}}^{\mathfrak{u}} \int_{\mathfrak{c}}^{\mathfrak{u}}\left(\mathfrak{u}^{s+1}-v^{s+1}\right)^{\frac{k}{\mathfrak{h}}-1}\left(\mathfrak{u}^{s+1}-m^{s+1}\right)^{\frac{k}{\mathfrak{h}}-1} \\
& \leqslant\|g\|_{\infty}^{2}\left[\frac{(s+1)^{1-\kappa}\left(u^{s+1}-c^{s+1}\right)^{\kappa}}{\Gamma(\kappa+1)}+\mathcal{R}_{c, \mathfrak{h}}^{\lambda, s}\left[u^{2 s+2}\right]\right. \\
& \left.+\frac{(s+1)^{1-\lambda}\left(u^{s+1}-c^{s+1}\right)^{\lambda}}{\Gamma(\lambda+1)}+\mathcal{R}_{c, h}^{k, s}\left[u^{2 s+2}\right]-2\left({ }_{+} \mathcal{R}_{c, \mathfrak{h}}^{k, s}[u]\right)\left({ }_{+} \mathcal{R}_{c, \mathfrak{h}}^{\lambda, s}[u]\right)\right] .
\end{aligned}
$$

Consequently, this with equation (2.10) proves the part (i) of the result.

To establish (ii), we will make use of the fact that

$$
\sup _{v, m \in[c, u]}\left|\left(v^{s+1}-m^{s+1}\right)\right|^{2}=\left(u^{s+1}-c^{s+1}\right)^{2}
$$


and get

$$
\frac{(s+1)^{2-\frac{k+\lambda}{\mathfrak{h}}}}{\mathfrak{h}^{2} \Gamma(\kappa) \Gamma(\lambda)} \int_{\mathfrak{c}}^{\mathfrak{u}} \int_{\mathfrak{c}}^{\mathfrak{u}}\left(u^{s+1}-v^{s+1}\right)^{\frac{k}{\mathfrak{h}}-1}\left(u^{s+1}-m^{s+1}\right)^{\frac{k}{\mathfrak{h}}-1} \leqslant\left(u^{s+1}-c^{s+1}\right)^{2}\left(+\mathcal{R}_{c, \mathfrak{h}}^{\kappa, s}[u]\right)\left(+\mathcal{R}_{c, \mathfrak{h}}^{\lambda, s}[u]\right) .
$$

Hence, this equation along with (2.10) proves the part (ii), i.e., (2.8) is established.

Deduction 2.6. By choosing $\mathrm{c}=\mathrm{d}$ of Theorem 2.5, we receive Theorem 2.1.

Theorem 2.7. Let $X$ be a r.v. having p.d.f. $\mathrm{g}:[\mathrm{c}, \mathrm{d}] \rightarrow \mathbf{R}^{+}$. Then

$$
+\mathcal{R}_{c, \mathfrak{h}}^{k, s}[g(u)] \sigma_{X, k, \mathfrak{h}}^{2}(u)-\left(E_{X-E(X), k}(u)\right)^{2} \leqslant \frac{1}{4}\left(d^{s+1}-c^{s+1}\right)^{2}\left(+\mathcal{R}_{c, \mathfrak{h}}^{k, s}[u]\right)^{2}
$$

for every $\mathrm{c}<\mathrm{u} \leqslant \mathrm{d}, \mathrm{k} \geqslant 0$ and $\mathrm{s} \geqslant 0$.

Proof. From Theorem 3.1 of [23], one can write

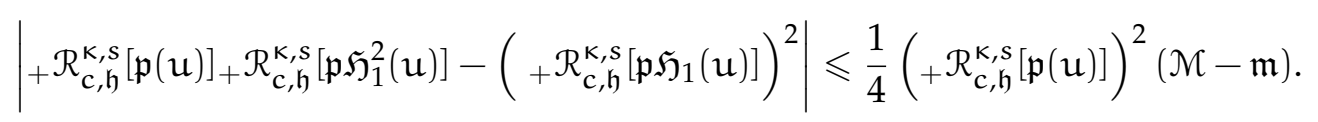

Now for $u \in[c, d]$, we choose $\mathfrak{p}(u)=g(u)$ and $\mathfrak{H}_{1}(u)=u^{s+1}-E(X)$, then $\mathcal{M}=d^{s+1}-E(X)$ and $\mathfrak{m}=$ $c^{s+1}-E(X)$. Thus, from (2.11), we can have

$$
\begin{aligned}
0 & \leqslant{ }_{+} \mathcal{R}_{c, \mathfrak{h}}^{k, s}[g(u)]_{+} \mathcal{R}_{c, \mathfrak{h}}^{k, s}\left[g(u)\left(u^{s+1}-E(X)\right)^{2}\right]-\left({ }_{+} \mathcal{R}_{c, \mathfrak{h}}^{k, s}\left[g(u)\left(u^{s+1}-E(X)\right)^{2}\right]\right)^{2} \\
& \leqslant \frac{1}{4}\left({ }_{+} \mathcal{R}_{c, \mathfrak{h}}^{k, s}[g(u)]^{2}\left(d^{s+1}-c^{s+1}\right)\right)^{2} .
\end{aligned}
$$

This yields that

$$
+\mathcal{R}_{c, \mathfrak{h}}^{\mathrm{k}, \mathrm{s}}[\mathrm{g}(\mathrm{u})] \sigma_{X, \mathrm{k}, \mathfrak{h}}^{2}(\mathrm{u})-\left(\mathrm{E}_{X-\mathrm{E}(X), \mathrm{k}}(\mathrm{u})\right)^{2} \leqslant \frac{1}{4}\left(\mathrm{~d}^{\mathrm{s}+1}-\mathrm{c}^{\mathrm{s}+1}\right)^{2}\left(+\mathcal{R}_{\mathrm{c}, \mathfrak{h}}^{\mathrm{k}, \mathrm{s}}[\mathrm{u}]\right)^{2}
$$

This completes the proof.

Now choosing $u=d$, we have following Corollary.

Corollary 2.8. For a p.d.f. g of r.v. $X$, we have

$$
\frac{\left(d^{s+1}-c^{s+1}\right)^{\frac{k}{h}-1}}{\Gamma(k)} \sigma_{X, k, \mathfrak{h}}^{2}(u)-\left(E_{X-E(X), \kappa}(u)\right)^{2} \leqslant \frac{1}{4 \Gamma^{2}(\kappa)}\left(d^{s+1}-c^{s+1}\right)^{2 \kappa}
$$

for $\mathrm{k} \geqslant 0$ and $\mathrm{s} \geqslant 0$.

Deduction 2.9. By choosing $\mathrm{k}=1=\mathfrak{h}$ in Corollary 2.8 we have Theorem 2 of [4].

Theorem 2.10. Let $X$ be a r.v. having p.d.f. $g:[c, d] \rightarrow \mathbf{R}^{+}$. Then

$$
\begin{gathered}
{ }_{+} \mathcal{R}_{c, \mathfrak{h}}^{k, s}[g(u)] \sigma_{X, \lambda}^{2}(u)+{ }_{+} \mathcal{R}_{c, \mathfrak{h}}^{\lambda, s}[g(u)] \sigma_{X, k, \mathfrak{h}}^{2}(u)+2\left(c^{s+1}-E(X)\right)\left(d^{s+1}-E(X)\right)_{+} \mathcal{R}_{c, \mathfrak{h}}^{k, s}[g(u)]_{+} \mathcal{R}_{c, \mathfrak{h}}^{\lambda, s}[g(u)] \\
\leqslant\left(c^{s+1}+d^{s+1}-2 E(X)\right)\left({ }_{+} \mathcal{R}_{c, \mathfrak{h}}^{k, s}[g(u)]\left(E_{X-E}(X), \lambda(u)\right)+{ }_{+} \mathcal{R}_{c, \mathfrak{h}}^{d, s}[g(u)]\left(E_{X-E(X), c}(u)\right)\right)
\end{gathered}
$$

for every $c<u \leqslant d, k \geqslant 0, \lambda \geqslant 0$ and $s \geqslant 0$. 
Proof. From Theorem 2.5 of [23], we can write

$$
\begin{aligned}
& {\left[{ }_{+} \mathcal{R}_{\mathbf{c}, \mathfrak{h}}^{\kappa, s}[\mathfrak{p}(\mathfrak{u})]_{+} \mathcal{R}_{\mathbf{c}, \mathfrak{h}}^{\lambda, \mathbf{s}}\left[\mathfrak{p} \mathfrak{H}_{1}^{2}(\mathfrak{u})\right]+{ }_{+} \mathcal{R}_{\mathbf{c}, \mathfrak{h}}^{\lambda, \mathbf{s}}[\mathfrak{p}(\mathfrak{u})]_{+} \mathcal{R}_{\mathfrak{c}, \mathfrak{h}}^{\kappa, s}\left[\mathfrak{p} \mathfrak{H}_{1}^{2}(\mathfrak{u})\right]\right.} \\
& \left.-2+\mathcal{R}_{c, \mathfrak{h}}^{k, s}\left[\mathfrak{p} \mathfrak{H}_{1}(u)\right]+\mathcal{R}_{c, \mathfrak{h}}^{\lambda, s}\left[\mathfrak{p} \mathfrak{H}_{1}(u)\right]\right]^{2} \\
& \leqslant\left[\left(\mathcal{M}_{+} \mathcal{R}_{c, \mathfrak{h}}^{k, s}[\mathfrak{p}(\mathfrak{u})]-{ }_{+} \mathcal{R}_{\mathfrak{c}, \mathfrak{h}}^{k, s}\left[\mathfrak{p} \mathfrak{H}_{1}(u)\right]\right)\left({ }_{+} \mathcal{R}_{c, \mathfrak{h}}^{\lambda, s}\left[\mathfrak{p} \mathfrak{H}_{1}(u)\right]-\mathfrak{m}_{+} \mathcal{R}_{c, \mathfrak{h}}^{\lambda, s}[\mathfrak{p}(u)]\right)\right. \\
& \left.+\left({ }_{+} \mathcal{R}_{\mathfrak{c}, \mathfrak{h}}^{\lambda, s}\left[\mathfrak{p} \mathfrak{H}_{1}(\mathfrak{u})\right]-\mathfrak{m}_{+} \mathcal{R}_{c, \mathfrak{h}}^{\lambda, s}[\mathfrak{p}(\mathfrak{u})]\right)\left(\mathcal{M}_{+} \mathcal{R}_{\mathfrak{c}, \mathfrak{h}}^{\lambda, s}[\mathfrak{p}(\mathfrak{u})]-{ }_{+} \mathcal{R}_{c, \mathfrak{h}}^{\lambda, s}\left[\mathfrak{p} \mathfrak{H}_{1}(\mathfrak{u})\right]\right)\right]^{2} .
\end{aligned}
$$

Choosing $\mathfrak{p}(\mathfrak{u})=g(u)$ and $\mathfrak{H}_{1}(\mathfrak{u})=v^{s+1}-E(X)$ in (2.12) yields

$$
\begin{aligned}
& {\left[{ }_{+} \mathcal{R}_{c, \mathfrak{h}}^{k, s}[g(u)]_{+} \mathcal{R}_{c, \mathfrak{h}}^{\lambda, s}\left[g(u)\left(u^{s+1}-E(X)\right)^{2}\right]+{ }_{+} \mathcal{R}_{c, \mathfrak{h}}^{\lambda, s}\left[g(u){ }_{+} \mathcal{R}_{c, \mathfrak{h}}^{k, s}\left[g(u)\left(u^{s+1}-E(X)\right)^{2}\right]\right.\right.} \\
&\left.-2{ }_{+} \mathcal{R}_{c, \mathfrak{h}}^{k, s}\left[g(u)\left(u^{s+1}-E(X)\right)\right]+\mathcal{R}_{c, \mathfrak{h}}^{\lambda, s}\left[g(u)\left(u^{s+1}-E(X)\right)\right]\right]^{2} \\
& \leqslant {\left[\mathcal{M}_{+} \mathcal{R}_{c, \mathfrak{h}}^{k, s}[g(u)]-{ }_{+} \mathcal{R}_{c, \mathfrak{h}}^{k, s}\left[g(u)\left(u^{s+1}-E(X)\right)\right]\right.} \\
& \times\left({ }_{+} \mathcal{R}_{c, \mathfrak{h}}^{\lambda, s}\left[g(u)\left(u^{s+1}-E(X)\right)\right]-m_{+} \mathcal{R}_{c, \mathfrak{h}}^{\lambda, s}[g(u)]\right) \\
& \times\left({ }_{+} \mathcal{R}_{c, \mathfrak{h}}^{k, s}\left[g(u)\left(u^{s+1}-E(X)\right)\right]-m_{+} \mathcal{R}_{c, \mathfrak{h}}^{k, s}[g(u)]\right) \\
&\left.\times\left(M_{+} \mathcal{R}_{c, \mathfrak{h}}^{\lambda, s}[g(u)]-{ }_{+} \mathcal{R}_{c, \mathfrak{h}}^{\lambda, s}\left[g(u)\left(u^{s+1}-E(X)\right)\right]\right)\right]^{2} .
\end{aligned}
$$

Now from (2.10) and (2.13) and using the fact that the left hand side of (2.10) is positive, we see

$$
\begin{aligned}
&{ }_{+} \mathcal{R}_{c, \mathfrak{h}}^{k, s}[g(u)]_{+} \mathcal{R}_{c, \mathfrak{h}}^{\lambda, s}\left[g(u)\left(u^{s+1}-E(X)\right)^{2}\right]+{ }_{+} \mathcal{R}_{c, \mathfrak{h}}^{\lambda, s}\left[g(u){ }_{+} \mathcal{R}_{c, \mathfrak{h}}^{k, s}\left[g(u)\left(u^{s+1}-E(X)\right)^{2}\right]\right. \\
&-2{ }_{+} \mathcal{R}_{c, \mathfrak{h}}^{k, s}\left[g(u)\left(u^{s+1}-E(X)\right)\right]+\mathcal{R}_{c, \mathfrak{h}}^{\lambda, s}\left[g(u)\left(u^{s+1}-E(X)\right)\right] \\
& \leqslant \mathcal{M}_{+} \mathcal{R}_{c, \mathfrak{h}}^{k, s}[g(u)]-{ }_{+} \mathcal{R}_{c, \mathfrak{h}}^{k, s}\left[g(u)\left(u^{s+1}-E(X)\right)\right]\left({ }_{+} \mathcal{R}_{c, \mathfrak{h}}^{\lambda, s}\left[g(u)\left(u^{s+1}-E(X)\right)\right]-m_{+} \mathcal{R}_{c, \mathfrak{h}}^{\lambda, s}[g(u)]\right) \\
& \times\left({ }_{+} \mathcal{R}_{c, \mathfrak{h}}^{k, s}\left[g(u)\left(u^{s+1}-E(X)\right)\right]-m_{+} \mathcal{R}_{c, \mathfrak{h}}^{k, s}[g(u)]\right) \\
& \times\left(M_{+} \mathcal{R}_{c, \mathfrak{h}}^{\lambda, s}[g(u)]-{ }_{+} \mathcal{R}_{c, \mathfrak{h}}^{\lambda, s}\left[g(u)\left(u^{s+1}-E(X)\right)\right]\right) .
\end{aligned}
$$

This yields us

$$
\begin{aligned}
& { }_{+} \mathcal{R}_{c, \mathfrak{h}}^{\mathrm{k}, \mathrm{s}}[\mathrm{g}(\mathrm{u})]_{+} \mathcal{R}_{\mathrm{c}, \mathfrak{h}}^{\lambda, \mathrm{s}}\left[\mathrm{g}(\mathrm{u})\left(\mathrm{u}^{\mathrm{s}+1}-\mathrm{E}(X)\right)^{2}\right]+{ }_{+} \mathcal{R}_{\mathrm{c}, \mathfrak{h}}^{\lambda, \mathrm{s}}[\mathrm{g}(\mathrm{u})]_{+} \mathcal{R}_{\mathrm{c}, \mathfrak{h}}^{\mathrm{k}, \mathrm{s}}\left[\mathrm{g}(\mathrm{u})\left(\mathrm{u}^{\mathrm{s}+1}-\mathrm{E}(X)\right)^{2}\right] \\
& \leqslant \mathcal{M}\left({ }_{+} \mathcal{R}_{\mathrm{c}, \mathfrak{h}}^{\kappa, s}[\mathrm{~g}(\mathrm{u})]\left(\mathrm{E}_{X-\mathrm{E}(X), \lambda}(\mathrm{u})\right)+{ }_{+} \mathcal{R}_{\mathrm{c}, \mathfrak{h}}^{\lambda, \mathrm{s}}\left[\mathrm{g}(\mathrm{u})\left(\mathrm{E}_{X-\mathrm{E}(X), \mathrm{k}}(\mathrm{u})\right)\right]\right) \\
& +\mathfrak{m}\left({ }_{+} \mathcal{R}_{c, \mathfrak{h}}^{k, s}[g(u)]\left(E_{X-E}(X), \lambda(u)\right)++\mathcal{R}_{c, \mathfrak{h}}^{\lambda, s}\left[g(u)\left(E_{X-E}(X), k(u)\right)\right]\right) .
\end{aligned}
$$

Using this in the values of $\mathcal{M}$ and $\mathfrak{m}$ from Theorem 2.7 and by simple calculations we get the result.

\section{Acknowledgment}

We sincerely appreciate all valuable comments and suggestions raised by the reviewers, that helped us to improve the quality of the manuscript. 


\section{References}

[1] G. Anastassiou, M. R. Hooshmandasl, A. Ghasemi, F. Moftakharzadeh, Montgomery identities for fractional integrals and related fractional inequalities, J. Inequal. Pure Appl. Math., 10 (2009), 1-6. 1

[2] R. Aziz, C. K. Verma, N. Srivastava, A novel approach for dimension reduction of microarray, Comput. Biol. Chem., 71 (2017), 161-169. 1

[3] R. Aziz, C. K. Verma, N. Srivastava, Artificial Neural Network Classification of High Dimensional Data with Novel Optimization Approach of Dimension Reduction, Ann. Data. Sci., 5 (2018), 615-635. 1

[4] N. S. Barnett, P. Cerone, S. S. Dragomir, J. Roumeliotis, Some inequalities for the expectation and variance of a random variable whose PDF is n-time differentiable, J. Inequal. Pure Appl. Math., 1 (2000), 13 pages. 2.3, 2.4, 2.9

[5] N. S. Barnett, S. S. Dragomir, R. P. Agarwal, Some inequalities for probability, expectation, and variance of random variables defined over a finite interval, Comput. Math. Appl., 43 (2002), 1319-1357. 1

[6] S. Belarbi, Z. Dahmani, On some new fractional integral inequalities, J. Inequal. Pure Appl. Math., 10 (2009), 5 pages.

[7] R. L. Blutzer, P. J. Torvik, On the fractional calculus model of viscoelastic behaviour, J. Rheol., 30 (1996), 133-155. 1

[8] Z. Dahmani, Fractional integral inequalities for continuous random variables, Malaya J. Mat., 2 (2014), 172-179. 1

[9] D. Fathima, A. H. Ganie, A. Antesar, Certain sequence spaces using $\Delta$-operator, Adv. Stud. Contemp. Math. (Kyungshang), 30 (2020), 17-27. 1

[10] A. H. Ganie, A. Aldawoud, Certain sequence spaces using $\Delta$-operator, Adv. Stud. Contemp. Math., 30 (2020), 17-27.

[11] M. A. Hammad, A. Awad, R. Khalil, E. Aldabbas, Fractional distributions and probability density functions of random variables generated using FDE, J. Math. Comput. Sci., 10 (2020), 522-534. 1

[12] T. Jonsson, J. Yngvason, Waves and Distributions, World Scientific, New Jersey, (1995).

[13] A. A. Kilbas, H. M. Srivastava, J. J. Trujillo, Theory and Applications of Fractional Differential Equations, Elsevier, Amsterdam, (2006).

[14] P. Kumar, Moments inequalities of a random variable defined over a finite interval, J. Inequal. Pure Appl. Math., 3 (2002), 11 pages. 1

[15] P. A. Naik, K. M. Owolabi, M. Yavuz, J. Zu, Chaotic dynamics of a fractional order HIV-1 model involving AIDS-related cancer cells, Chaos Solitons Fractals, 140 (2020), 13 pages. 1

[16] P. A. Naik, J. Zu, K. M. Owolabi, Global Dynamics of a fractional order model for the transmission of HIV Epidemic with optimal control, Chaos Solitons Fractals, 138 (2020), 24 pages. 1, 1.1

[17] P. A. Naik, J. Zu, K. M. Owolabi, Modeling the Mechanics of Viral Kinetics under Immune Control during Primary Infection of HIV-1 with Treatment in Fractional Order, Phys. A, 545 (2020), 19 pages. 1.1

[18] M. Niezgoda, New bounds for moments of continuous random variables, Comput. Math. Appl., 60 (2010), 3130-3138. 1

[19] F. Qi, A.-J. Li, W.-Z. Zhao, D.-W. Niu, J. Cao, Extensions of several integral inequalities, J. Inequal. Pure Appl. Math., 7 (2006), 6 pages. 1

[20] S. Salaş, Y. Erdaş, T. Toplu, E. Set, On Some Generalized Fractional Integral Inequalities for p-Convex Functions, Fractal Fract., 3 (2019), 1-9. 1

[21] S. G. Samko, A. A. Kilbas, O. I. Marichev, Fractional Integrals and Derivatives, Theory and Applications, Gordon and Breach Science Publishers, Switzerland, (1993). 1.1

[22] R. Sharma, S. Devi, G. Kapoor, S. Ram, N. S. Barnett, A brief note on some bounds connecting lower order moments for random variables defined on a finite interval, Int. J. Theor. Appl. Sci., 1 (2009), 83-85. 1

[23] M. Tomar, S. Maden, E. Set, ( $k, s)$-Riemann-Liouville fractional integral inequalities for continuous random variables, Arab. J. Math., 6 (2017), 55-63. 2, 2

[24] M. I. Troparevsky, S. A. Seminara, M. A. Fabio, A Review on Fractional Differential Equations and a Numerical Method to Solve Some Boundary Value Problems, Nonlinear Syst., (2019). 1

[25] H. Yildirim, Z. Kirtay, Ostrowski inequality for generalized fractional integral and related inequalities, Malaya J. Mat., 2 (2014), 322-329. 1.2, 1.3, 1.5 